\title{
A reply to the letter to the editor regarding "microstructural changes of the corticospinal tract in idiopathic normal pressure hydrocephalus: a comparison of diffusion tensor and diffusional kurtosis imaging"
}

\author{
Masaaki Hori • Atsushi Nakanishi • Shigeki Aoki
}

Received: 14 August 2013 / Accepted: 15 August 2013 / Published online: 29 August 2013

(C) The Author(s) 2013. This article is published with open access at Springerlink.com

Dear Sir,

We read with interest the letter concerning our article "Microstructural changes of the corticospinal tract in idiopathic normal pressure hydrocephalus: a comparison of diffusion tensor and diffusional kurtosis imaging." The correspondents noted that diffusion metrics such as fractional anisotropy (FA) and apparent diffusion coefficient (ADC) depend on the combination of data (i.e., different $b$ values) and theory (Gaussian or non-Gaussian). We quite agree with their opinion that FA of diffusion tensor imaging (DTI) should be calculated from tensor model usually from $b=0$ and 1,000 . Our description might lead the readers to misunderstanding.

We think that our description of the section "Tractography and tract-specific analysis of CST" was unclear because we indeed calculated ADC and FA from a portion of the diffusional kurtosis imaging (DKI) data based on $b$ values of 0 and $1,000 \mathrm{~s} / \mathrm{mm}^{2}$ using the conventional model. These calculated values were then used as the estimates for ADC and FA. We also recognize that the conventional DTI metrics for clinical studies should be estimated by using the same $b$ values of 0 and $1,000 \mathrm{~s} / \mathrm{mm}^{2}$, as in the many reports in the clinical DTI literatures. In other studies concerning DKI, we explicitly stated that the DTI metrics used for comparison with DKI are calculated using the data obtained from $b$ values of 0 and $1,000 \mathrm{~s} / \mathrm{mm}^{2}$ and a mono-exponential fit [1-3]. Moreover, although we did not provide the details in this paper, our software for DTI and DKI calculation (dTV.II.FZRx; Image Computing and Analysis Laboratory, Department of Radiology, The University of Tokyo Hospital, Japan) [4] has

This comment refers to the article available at 10.1007/s00234-013-1271-5.

M. Hori $(\bowtie) \cdot$ A. Nakanishi $\cdot$ S. Aoki

Department of Radiology, Juntendo University School of Medicine,

2-1-1 Hongo Bunkyo-ku, Tokyo 113-8421, Japan

e-mail: mahori@juntendo.ac.jp the ability to calculate DTI metrics such as FA and ADC on the basis of the mono-exponential model by using user-specified $b$ value data when performing DKI calculations.

The letter, which suggests that differences between the diffusion metrics derive from differences in parameters or analysis theories, is still of great value. We agree that in DKI or other diffusion-related studies that employ non-conventional signal-fitting procedures, the authors should clarify the type of method and data used for calculating the diffusion metrics.

Conflict of interest We declare that we have no conflict of interest.

Open Access This article is distributed under the terms of the Creative Commons Attribution License which permits any use, distribution, and reproduction in any medium, provided the original author(s) and the source are credited.

\section{References}

1. Kamagata K, Tomiyama H, Motoi Y, Kano M, Abe O, Ito K, Shimoji K, Suzuki M, Hori M, Nakanishi A, Kuwatsuru R, Sasai K, Aoki S, Hattori N (2013) Diffusional kurtosis imaging of cingulate fibers in Parkinson disease: comparison with conventional diffusion tensor imaging. Magn Reson Imaging. doi:10.1016/j.mri.2013.06.009

2. Yoshida M, Hori M, Yokoyama K, Fukunaga I, Suzuki M, Kamagata K, Shimoji K, Nakanishi A, Hattori N, Masutani Y, Aoki S (2013) Diffusional kurtosis imaging of normal-appearing white matter in multiple sclerosis: preliminary clinical experience. Jpn J Radiol 31(1):50-55. doi:10.1007/s11604-012-0147-7

3. Hori M, Fukunaga I, Masutani Y, Nakanishi A, Shimoji K, Kamagata K, Asahi K, Hamasaki N, Suzuki Y, Aoki S (2012) New diffusion metrics for spondylotic myelopathy at an early clinical stage. Eur Radiol 22(8):1797-1802. doi:10.1007/s00330-012-2410-9

4. Hori M, Fukunaga I, Masutani Y, Taoka T, Kamagata K, Suzuki Y, Aoki S (2012) Visualizing non-Gaussian diffusion: clinical application of q-space imaging and diffusional kurtosis imaging of the brain and spine. Magn Reson Med Sci 11(4):221-233 